\title{
Detect the Optimal Skyline Product Combinations Under Price Promotion
}

\author{
PS Naveen Kumar, Ch.Tirupathi Rayudu \\ Assistant professor Dept.of MCA, St.Ann's College of Engineering \& Technology, Chirala. \\ PG Student, Dept.of MCA, St.Ann's College of Engineering \& Technology, Chirala.
}

\begin{abstract}
These days, with the improvement of web-based business, a developing number of clients go out on the town to shop on the web. To discover alluring items from internet shopping commercial centers, the horizon inquiry is a valuable device which offers all the more intriguing and ideal decisions for clients. The horizon inquiry and its variations have been widely examined. Be that as it may, to the best of our insight, they have not considered the necessities of clients in certain reasonable application situations. As of late, web-based shopping commercial centers as a rule hold some cost advancement battles to draw in clients and increment their buy expectation. Thinking about the necessities of clients in this down to earth application situation, we are worried about item determination under value advancement. We define a compelled ideal item blend (COPC) issue. It means to discover the horizon item mixes which both meet a client's eagerness to pay and bring the most extreme rebate rate. The COPC issue is critical to offer incredible choice help for clients under value advancement, which is guaranteed by a client consider. To process the COPC issue adequately, we initially propose a two-rundown correct (TLE) calculation. The COPC issue is turned out to be NP-hard, and the TLE calculation isn't versatile in light of the fact that it needs to process an exponential number of item mixes. Furthermore, we structure a lower bound estimated (LBA) calculation that has ensured about the precision of the outcomes and a gradual voracious (IG) calculation that has great execution. The examination results show the productivity and adequacy of our proposed calculations.
\end{abstract}

Keywords: - Data the executives, value advancement, horizon inquiry, NP-hard.

\section{INTRODUCTION}

With the advancement of internet business, a developing number of clients go out on the town to shop online in light of the fact that it spares time and exertion. Be that as it may, it generally contraries to desires for clients. This is on the grounds that they may need to get one decision among a large number of items. To encourage clients distinguish appealing items, a horizon question is as a matter of fact a typical and compelling approach. As indicated by the meaning of the horizon inquiry [1], an item which isn't overwhelmed by some other item is said to be a horizon item or it is in the horizon. The items in the horizon are the most ideal tradeoffs between every one of the variables that clients care about. The horizon inquiry is helpful in distinguishing appealing items. In Jingdong and Alibaba's TaobaoMall which are the most acclaimed internet shopping centers in China, there are numerous online stores that work in one class of items, for example, red wine, watches, $\mathrm{TV}, \mathrm{PC}$, to give some examples. Amid the ends of the week or occasions, these stores for the most part hold some value advancement crusades to support utilization. Under the value advancement battles of these stores, a client could choose an ideal item mix independent from anyone else. In addition, the client is normal to take an interest in collaboration with his families or companions for gathering purchasing. The present value advancement crusades can be characterized into two classifications because of whether items can be picked freely. The principal classification, in particular, autonomous item determination, incorporates the crusades, for example, "get one item and get another item for nothing" and "25\% rebate for two pics" and so forth. Under these crusades, clients can select the items satisfying their needs autonomously and specifically, and horizon inquiries could offer ground-breaking choice help. The second class, to be specific, subordinate item determination, comprises of the battles, for example, "get $\$ 60$ off each $\$ 200$ buy" and "\$100 coupon each $\$ 500$ buy" and so forth. In these situations, clients dependably hope to choose items which are appealing and bring the bestadvantage. Besides, it needs to mull over the client's readiness to pay which is an imperative issue that influences the client's buying conduct. The horizon question is ground-breaking to process the horizon items that have a solid intrigue to clients. In any case, it is insufficient to enable clients to choose horizon item blends with the best advantage. Thinking about the necessities of clients in this viable application 


\section{International Journal of Research in Advent Technology, Vol.7, No.2, February 2019 E-ISSN: 2321-9637 Available online at $w w w . i j r a t . o r g$}

situation, we are worried about another issue of distinguishing ideal item mixes under value advancement battles. In this paper, we center around the needy item choice battles that are considerably more famous yet entangled with correlation with the free item determination crusades. Expect that Jingdong offers a value advancement battle which is "get \$60 off each $\$ 200$ buy" (we will utilize this value advancement crusade in all the rest of the precedents). We take three qualities of each wine, which are class, laud degree, and unique cost, into record. The French wines are typically separated into four classes which are

1. Vin de France (VDF),

2. Vin de Pays (VDP),

3. VinDelimitesdeQualiteSuperieure (VDQS), and

4. Nickname d'OrigineProtegee (AOP). Without loss of simplification, for the two qualities, class and acclaim degree, of the French wines, extensive qualities are viewed as ideal over little ones. At the first cost, little esteem is superior to expansive one. In this paper, we measure the item blends by their rebate rates, and spotlight on the most proficient method to consolidate homogenous items under value advancement battles. Furthermore, now and again, clients may need to choose items from various classes, this is out of the degree in this paper. In the writing, the firmly related investigates to our concern are assemble horizon questions and horizon inquiries under limitations. In any case, they can't be connected to our concern specifically as investigated in Section 2. To the best of our insight, we examine item choice under value advancement without precedent for the writing. Our commitments are quickly condensed as pursues.

- We devise the COPC issue. This issue plans to discover horizon item mixes which meet a client's installment eagerness and bring the greatest markdown rate. We demonstrate the COPC issue is NP-hard.

- We propose a correct calculation, in particular two rundown correct calculation, for the COPC issue. Also, we plan a lower bound estimated calculation, which has ensure about the exactness of the outcomes. To improve execution, we build up the steady insatiable calculation for the COPC issue.

- We acquaint how with stretch out the proposed ways to deal with handle the relating issue under other value advancements and talk about two variations of the COPC issue by considering distinctive client requests.

- We direct a little client concentrate to confirm the huge of our COPC issue and play out a broad test concentrate to clear up the viability and proficiency of all the proposed calculations.

\section{RELATED WORK}

As an imperative information the board administrator the horizon question and its variations has gotten an incredible consideration in the writing In our COPC issue, it registers the ideal horizon item blends with a requirement, which is the client's ability to pay. The firmly related issues are assembling horizon inquiries and horizon questions under limitations, and the related works are surveyed in this segment.

\subsection{Gathering Skyline Queries:}

The horizon question plans to restore the focuses that are not commanded by some other point [1]. In any case, a large portion of the works about the horizon question simply examine singular focuses, and they are unseemly to numerous applications that call for investigation of gatherings of various focuses. Roused by this, bunch horizon questions are produced and gave careful consideration. In a large portion of the gathering horizon questions, ideal gatherings are registered by the strength connection between relating total based purposes of various gatherings. Su et al. [3] figured best k combinatorial horizon question (k-CSQ). It restores those combinatorial horizon tuples whose total qualities for a specific characteristic are greatest. Since just the primary $\mathrm{k}$ mixes are required, the $\mathrm{k}-\mathrm{CSQ}$ inquiry process can be streamlined [4]. Chung et al. [4] broadened the conventional horizon inquiries and detailed a combinatorial horizon inquiry, in particular CSQ, which is to locate the remarkable horizon mixes. Im et al. [5] considered the gathering horizon question which depends on the predominance connection between the gatherings of a similar size. The strength relationship is checked by the total estimations of qualities. Magnani et al. [26] presented total horizons, where the horizon fills in as a separating predicate on sets of records. The total horizon questions consolidate the functionalities $\mathrm{f}$ two essential database administrators, horizon and gathering by. Zhang et al. [7] concentrated on a novel issue of gatherings of $\mathrm{k}$ tuples, which are not overwhelmed by some other gathering of equivalent size, in view of total based gathering strength relationship. They additionally distinguished two enemy of monotonic properties to sift through competitor gatherings. Kumar et al. [6] illustrate the interventions and exploit examine cycles enacted more a period of five years in alliance with the firm, which ensued in a norm SPE skeleton that catered to both the societal and scientific needs of the firm's 


\section{International Journal of Research in Advent Technology, Vol.7, No.2, February 2019 E-ISSN: 2321-9637 Available online at www.ijrat.org}

scattered teams. Institutionalizing the process ripeness skeleton got stalled primarily because the SPE initiatives were superficial by product line managers as a apparatus for exercising wider controls by the firm's top management. The accomplishment apparatus was afterward altered to co-opt product line managers, which supplied to a wider espousal of the SPE skeleton. Insights that come out from our scrutiny of the firm's SPE crossing pertain to the amalgamation of the technical and social sights of software development, perpetuating course of action assortment all the way over the use of a multi-tiered, non - sketch come within reach of to SPE, the linkage among key process areas and project have power over modes, and the protagonist of SPE in aiding governmental learning. Wu et al. [2] inquired about the work which is like the gathering horizon calculation. They concentrated on an issue of making focused items which are not ruled by the items in the current market. Here each new item is created by brushing items from various source tables. To decrease the hunt space, it just joins the horizon items from source tables to create new items. What's more, they additionally displayed a methodology which isolates comparative items into gatherings and procedures them in general. In the gathering horizon inquiries previously mentioned, the total benefits of comparing traits are considered while checking the predominance connection between various gatherings. In any case, it is troublesome for clients to indicate a proper total capacity. In addition, it disregards numerous noteworthy gatherings that may contain nonskyline focuses [8]. Spurred by the issues which the gathering horizon questions confront, Liu et al. [8] displayed a Pareto assemble based horizon (G-horizon) inquiry which recovers Gskyline gatherings. These G-horizon bunches are not g-ruled by some other gathering of a similar size. They introduced a coordinated horizon diagram which catches the predominant relationship of tuples inside the primary $\mathrm{k}$ horizon layers. Besides, two heuristic calculations are intended to fathom the G-horizon question viably. As of late, Yu et al. [9] characterized the Multiple ple Horizon Layers, and exhibited two quick calculations to register the G-horizons because of the perception that horizon indicates contribute more horizon bunches contrasted with non-skyline focuses. The above methodologies for gathering horizon questions are unseemly for our concern. This is since they sift through the ideal gatherings by the predominance relationship, however the horizon item blends in our COPC issue are estimated by the most extreme rebate rate.

\subsection{Horizon Queries under Constraints}

Horizon question is a valuable apparatus to discover alluring items which offer all the more fascinating and best decisions for clients. In any case, the extent of the horizon inquiry results can't be controlled adaptably. In like manner, many research endeavors have been committed to battle with this issue. The current ways to deal with location this issue are produced to recognize $\mathrm{k}$ delegate horizons which have the greatest prevailing limit or the most extreme broadening. Lu et al. [10] were worried about the situation when the genuine cardinality of horizon results is not exactly the ideal outcome cardinality $\mathrm{k}$. They proposed another methodology, in particular horizon requesting, which frames a horizon-based dividing of a given dataset. At that point they connected a set-wide augmentation method, which is utilized to discover an item set commanding the biggest number of focuses, to process each parcel. Bai et al. concentrated on the best way to pick $\mathrm{k}$ agent horizons over information streams. Gao et al. [18] planned the most attractive horizon object inquiry which reports the most best $\mathrm{k}$ horizons dependent on another positioning paradigm. Lin et al. [12] considered the issue of choosing $\mathrm{k}$ horizon focuses with the end goal that the quantity of focuses, which are overwhelmed by something like one of these k horizons focuses, is boosted. Papadias et al. [11] proposed the K-overwhelming question which recovers $\mathrm{K}$ focuses commanding the biggest number of different focuses. This question does not really contain horizon focuses but rather has the benefits of both positioning inquiries and horizon questions, which are with the control on the span of the appropriate response set and without clients' endeavors to indicate positioning capacities. Chan et al. [15] detailed a best $\mathrm{k}$ positioning issue which recovers focuses showing up much of the time in the subspace horizons. Wan et al. [14] recognized the issue of discovering top-k beneficial items. Given a lot of bundles in the current market and a lot of potential new bundles, they needed to choose $\mathrm{k}$ new bundles to such an extent that the aggregate of the benefits of the chose bundles is expanded and each chosen bundle isn't overwhelmed by any bundle in the current market and any chosen new bundle. Lin et al. [13] proposed a k-most requesting items (k-MDP) finding issue that causes the organization to choose $\mathrm{k}$ items from the competitor items with the greatest anticipated number of the all out clients. In [20], we built up a best $\mathrm{k}$ most loved probabilistic item question. It is used to choose $\mathrm{k}$ items which can address the issues of a client set at the most extreme dimension. The delegate horizon questions 
previously mentioned just feature a few highlights which are security, scale invariance, broadening of the outcomes, and incomplete information of the record scoring capacity [16]. Magnani et al. [16] concentrated on the delegate horizon questions as far as both the importance and assorted variety of results. This delegate horizon question can fulfill every one of the highlights above. In addition, there are likewise some different ways to deal with abstain from returning numerous horizon inquiry results. In , Chen et al. contemplated an obliged horizon question. It first sifts through the focuses which don't fulfill extend requirements of every trait, and afterward forms the horizon question over the rest of the focuses.

\section{EXISTING SYSTEM}

The present value advancement crusades can be grouped into two classifications because of whether items can be picked autonomously. The main class, to be specific, autonomous item choice, incorporates the battles, for example, "get one item and get another item for nothing" and "25\% markdown for two pics" and so forth. Under these battles, clients can select the items satisfying their needs autonomously and specifically, and horizon questions could offer ground-breaking choice help. The second class, to be specific, subordinate item choice, comprises of the crusades, for example, "get $\$ 60$ off each \$200 buy" and "\$100 coupon each \$500 buy" and so on.

\subsection{Disadvantages}

Under these battles, clients can select the items fulfilling their needs freely and specifically.

\section{PROPOSED SYSTEM}

The clients dependably hope to choose items which are appealing and bring the best advantage. Also, it needs to mull over the client's eagerness to pay which is an essential issue that influences the client's acquiring conduct. The horizon inquiry is amazing to figure the horizon items that have a solid intrigue to clients. Nonetheless, it is lacking to help client's select horizon item mixes with the best advantage. Thinking about the prerequisites of clients in this down to earth application situation, we are worried about another issue of distinguishing ideal item blends under value advancement battles. In this, we center around the needy item determination battles that are significantly more mainstream however confounded with correlation with the autonomous item choice crusades.

\subsection{Preferred standpoint:}

This issue plans to discover horizon item mixes which meet a client's installment eagerness and bring the most extreme markdown rate. Horizon inquiries could offer ground-breaking choice help.

\subsection{System Modules:}

4.2.1The Constrained Optimal Product Combination (Copc) Problem:

In the COPC issue, it needs to figure the horizon items by the horizon inquiry which a helpful device for choice help is. The horizon question over every one of the credits may offer ascent to free some vital item blends.

\subsubsection{The Two List Exact issues:}

Because of the COPC issue is firmly identified with the subset entirety issue. Also, our COPC issue is substantially more confounded, and the methodologies for the subset issue can't be used to our concern specifically. In this, we build up the twolist calculation, which is an acclaimed calculation for the subset entirety issue and present a two rundown correct calculation for the COPC issue.

\subsubsection{The Lower Bound Approximation:}

Plan a lower headed inexact calculation for the COPC issue, The LBA calculation first expels every item $\mathrm{p}^{\prime} \in \mathrm{SP}$ whose genuine installment is bigger than WTP (Line 1). Line 2 instates a rundown $L$ with a set that contains a component " 0 ". From that point, the rundown $\mathrm{L}$ stores unique costs of competitor horizon item blends.

\section{CONCLUSION}

In this paper, we define the COPC issue to recover ideal horizon item mixes that fulfill the client's installment imperative and bring the greatest markdown rate. To handle the COPC issue, we propose a correct calculation, plan a rough calculation with a surmised bound, and build up a steady voracious calculation to help the execution. We direct a client concentrate to check the huge of our COPC issue. Furthermore, the test results on both genuine and manufactured datasets delineate the viability and productivity of the proposed calculations. This work opens to some encouraging bearings for future work. In the first place, notwithstanding mixes of homogeneous items, we will concentrate on the COPC issue over results of various classifications. From that point onward, in all actuality, the client's requests are broadening and individuation, and it is critical and intriguing to figure ideal item blends that fulfill diverse client needs, for 
example, spare or spend the most cash under their financial plans. To wrap things up, we could likewise investigate top $\mathrm{k}$ COPC issue that means to process $\mathrm{k}$ ideal item mixes because of client requests dependent on the work in.

\section{REFERENCES: -}

[1] D. Papadias, Y. Tao, G. Fu, and B. Seeger, "Progressive skylinecomputation in database systems," ACM Transactions on DatabaseSystems (TODS), vol. 30, no. 1, pp. 4182, 2005.

[2] X. Lin, Y. Yuan, Q. Zhang, and Y. Zhang, "Selecting stars: The kmost representative skyline operator," in Proc. 23th Int'l Conf. DataEng. (ICDE), pp. 86-95, IEEE, 2007.

[3] C.-Y. Lin, J.-L. Koh, and A. L. Chen, "Determining k-most demandingproducts with maximum expected number of total customers,"IEEE Trans. on Knowl. Data Eng, vol. 25, no. 8, pp. 1732-1747, 2013.

[4] Q. Wan, R.-W. Wong, and Y. Peng, "Finding topk profitableproducts," in Proc. 27th Int'l Conf. Data Eng. (ICDE), pp. 1055-1066,IEEE, 2011.

[5] C.-Y. Chan, H. Jagadish, K.-L. Tan, A. K. Tung, and Z. Zhang, "On high dimensional skylines," in Proc. Advances in DatabaseTechnology (EDBT), pp. 478-495, Springer, 2006.

[6] Maddali M.V.M. Kumar, "A Review on Software Process Enhancements of Product Development in Worldwide Scattered Governance", International Research Journal of Engineering \& Technology; Vol. - 03; Issue - 02; pp: 1111-1114; February 2016

[7] G. Xiao, K. Li, X. Zhou, and K. Li, "Efficient monochromaticand bichromatic probabilistic reverse top-k query processing foruncertain big data," Journal of Computer \& System Sciences, vol. 89,2016.

[8] Y. Gao, Q. Liu, L. Chen, G. Chen, and Q. Li, "Efficient algorithmsfor finding the most desirable skyline objects ," KnowledgeBasedSystems, vol. 89, no. C, pp. 250-264, 2015.

[9] M. Bai, J. Xin, G. Wang, and L. Zhang, "Discovering the $\mathrm{k}$ representativeskyline over a sliding window," IEEE Trans. on Knowl.Data Eng, vol. 28, no. 8, pp. 2041-2056, 2016.

[10] X. Zhou, K. Li, Y. Zhou, and K. Li, "Adaptive processing fordistributed skyline queries over uncertain data," IEEE Trans. OnKnowl. Data Eng, vol. 28, no. 2, pp. 371-384, 2016.

[11] L. Chen, B. Cui, and H. Lu, "Constrained skyline query processingagainst distributed data sites," IEEE Trans. on Knowl. Data Eng,vol. 23, no. 2, pp. 204-217, 2011.

[12] T. H. Cormen, Introduction to algorithms. MIT press, 2009.

[13] F. B. Chedid, "A note on developing optimal and scalable paralleltwo-list algorithms," in International Conference on Algorithms andArchitectures for Parallel Processing, pp. 148-155, 2012.

[14] C. A. A. Sanches, N. Y. Soma, and H. H. Yanasse, "Observations onoptimal parallelizations of two-list algorithm," Parallel Computing,vol. 36, no. 1, pp. 65-67, 2010.

[15] A. G. Ferreira, "A parallel time/hardware tradeoff t.h $=\mathrm{o}(2 / \mathrm{supn} / 2 /)$ for the knapsack problem," IEEE Trans. on Computers, vol. 40, no. 2, pp. 221-225, 1991.

[16] Y. Tao, X. Xiao, and J. Pei, "Efficient skyline and top-k retrievalin subspaces," IEEE Trans. on Knowl. Data Eng., vol. 19, no. 8,pp. 1072-1088, 2007.

[17] J.-L. Koh, C.-Y. Lin, and A. L. Chen, "Finding $k$ most favorite productsbased on reverse top- $t$ queries," The VLDB Journal, vol. 23,no. 4, pp. 541-564, 2014.

[18] M. S. Islam and C. Liu, "Know your customer: computing k-mostpromising products for targeted marketing," The VLDB Journal,vol. 25, no. 4, pp. 545-570, 2016.

[19] X. Zhou, K. Li, G. Xiao, Y. Zhou, and K. Li, "Top k favoriteprobabilistic products queries," IEEE Trans. on Knowl. Data Eng,pp. 2808-2821, 2016.

[20] Q. Wan, R. C.-W. Wong, I. F. Ilyas, M. T. O" zsu, and Y. Peng,"Creating competitive products," Proc. of the VLDB Endowment,vol. 2, no. 1, pp. 898-909, 2009.

[21] I.-F. Su, Y.-C. Chung, and C. Lee, "Top-k combinatorial skylinequeries," in Database Systems for Advanced Applications, pp. 7993,Springer, 2010.

[22] Y.-C. Chung, I.-F. Su, and C. Lee, "Efficient computation of combinatorialskyline queries," Information Systems, vol. 38, no. 3,pp. 369-387, 2013.

[23] H. Im and S. Park, "Group skyline computation," InformationSciences, vol. 188, pp. 151-169, 2012.

[24] M. Magnani and I. Assent, "From stars to galaxies: skyline querieson aggregate data," in Proc.16th Int'l Conf. on Extending DatabaseTechnology, pp. 477-488, ACM, 2013.

[25] M. Magnani, I. Assent, and M. L. Mortensen, "Taking the big picture:representative skylines 
International Journal of Research in Advent Technology, Vol.7, No.2, February 2019

E-ISSN: 2321-9637

Available online at www.ijrat.org

based on significance and diversity,"The $V L D B$

Journal, vol. 23, no. 5, pp. 795-815, 2014.

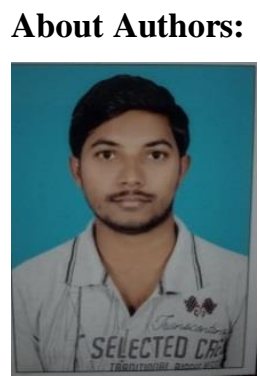

Ch.Tirupathi Rayudu is currently pursuing his MCA inMCA Department St.Ann's College of Engineering and Technology, Chirala, A.P. He received her Bachelor of Science from ANU.

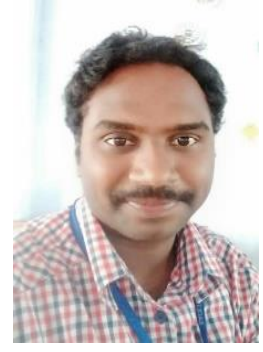

PS Naveen Kumar, MCA, Mtech(CSE) and pursuing Ph.D in Annamalai University. $\mathrm{He}$ is currently working as a Assistant Professor of MCA Department in St.Ann's College of Engineering \& Technology, Chirala-523187, A.P. He had 8 years of Experience and his interesting areas are DataMining and Signal Processing. 